\title{
Vortex nucleation and hysteresis phenomena in rotating Bose-Einstein condensates.
}

\author{
Juan J. García-Ripoll and Víctor M. Pérez-García \\ Departamento de Matemáticas, Escuela Técnica Superior de Ingenieros Industriales \\ Universidad de Castilla-La Mancha, 13071 Ciudad Real, Spain
}

(October 31, 2018)

\begin{abstract}
We study the generation of vortices in rotating Bose-Einstein condensates, a situation which has been realized in a recent experiment (K. W. Madison, F. Chevy, W. Wohlleben, J. Dalibard, Phys. Rev. Lett. 84806 (2000)). By combining a linear stability analysis with the global optimization of the nonlinear free energy functional, we study the regimes that can be reached in current experiments. We find a hysteresis phenomenon in the vortex nucleation due to the metastabilization of the vortexless condensate. We also prove that for a fast enough rotating trap the ground state of the condensate hosts one or more bent vortex lines.

PACS number(s): 03.75.Fi, 05.30.Jp, 67.57.De, 67.57.Fg
\end{abstract}

Vortices, or vortex lines we should rather say, constitute the most relevant topological defect in Physics. They consist on a twist of the phase of a wave function around an open line and they are typically associated to a rotation of a fluid whatever the fluid is made of (real fluids, optical fluids, quantum fluids, ...) [1]. Vortices are one of the means by which quantum systems acquire angular momentum and react to perturbations of the environment. They have already been predicted, observed and studied in the superfluid phase of ${ }^{4} \mathrm{He}$ and are indeed known to be the key to some important processes in these systems, such as dissipation, moments of inertia and breakdown of superfluidity. This is why extensive research on vortex generation, stability and dynamics has been conducted in the field of Bose-Einstein condensation (BEC) in the last years [2 5].

Vortices and other defects usually involve more energy than other equilibrium states, such as convex nodeless states. Therefore, in order to produce a vortex in a condensate, one must induce a transition from a uncondensed or condensed convex cloud to the desired state, by means of an external action such as a change of the confining potential.

For instance, in recent experiments performed by a group from the École Normale Supérieure (ENS) [6], vortices are created by rotating an elongated trap which is slightlty deformed along its transverse dimensions. After this preparation, the trap is switched off, and the condensate expands until vortices become directly observable.

In these experiments there are several controversial points which are: (i) Vortices first nucleate at a trap rotation speed or critical frequency, $\Omega_{1}$, larger than that required to stabilize a vortex line in the Thomas-Fermi theory, (ii) when seen from the top vortex cores seem partially filled and (iii) after the nucleation of the first vortex the angular momentum grows continuously with the rotation speed (not only with discontinuous jumps).

In this paper we reach a global view of the transitions between equilibrium states which are induced by the trap rotation, plus a simple explanation of the most contro- versial points. Our study consists of two parts. First we focus on the analysis of stationary states and discuss the stability properties of the simplest solutions, i.e. straight vortices, which allows us to get some insight on the problem. Our main result is that the metastabilization of a vortexless state induces hysteresis in the vortex nucleation process. In other words, the trap rotation speed must exceed a critical value, $\Omega_{M}$, to produce vortices, but that speed is well beyond the values, $\Omega_{1}, \Omega_{2}, \ldots$, which are required to make vortices energetically favorable. In the second part we find numerically the actual ground states of the condensate for different angular speeds. We will show that once the first vortex is nucleated the angular momentum grows almost continuously with the speed of the trap, while the ground state (GS) mutates into different deformed states.

Several other works have contributed to the theoretical description of the experimental "anomalies". First, Ref. in [7] the dynamics of vortex lines is studied analytically, and different modes are obtained which reflect the transverse tension of vortex lines. Next, in Ref. [8] the existence of these modes is confirmed numerically a relation between these modes and the large value of the critical angular speed of the ENS experiments is proposed. Finally in Ref. [9] the authors derive a condition for the efficiency of external perturbations when trying to induce a mechanical response in the condensate.

The model.- For most of current experiments it is an accurate approximation to use a zero temperature manybody theory of the condensate. In that limit the whole condensate is described by a single wavefunction $\psi(\mathbf{r}, t)$ ruled by a Gross-Pitaevskii equation (GPE) [10].

In Ref. [6] the trap is initially harmonic with axial symmetry, but then a laser is applied which deforms it and makes it rotate with uniform angular speed $\Omega$. On the mobile reference frame which rotates with the trap, the experiment is modeled by a modified GPE

$$
i \frac{\partial \psi}{\partial t}=\left[-\frac{1}{2} \triangle+V_{0}(\mathbf{r})+\frac{g}{2}|\psi|^{2}-\Omega L_{z}\right] \psi .
$$


Here $L_{z}=i\left(x \partial_{y}-y \partial_{x}\right)$ is the hermitian operator that represents the angular momentum along the z-axis and the effective trapping potential in given by

$$
V_{0}(\mathbf{r})=\frac{1}{2} \omega_{\perp}^{2}(1-\varepsilon) x^{2}+\frac{1}{2} \omega_{\perp}^{2}(1+\varepsilon) y^{2}+\frac{1}{2} \omega_{\perp}^{2} \gamma^{2} z^{2} .
$$

In Eq. (11) we have applied a convenient adimensionalization which uses the harmonic oscillator length, $a_{\perp}=\sqrt{\hbar / m_{R b} \omega_{\perp}}$ and period, $\tau=\omega_{\perp}^{-1}$. With these units the nonlinear parameter becomes $g=4 \pi a_{\mathrm{S}} / a_{\perp}$, where $a_{\mathrm{S}} \simeq 5.5 \mathrm{~nm}$ is the scattering length for ${ }^{87} \mathrm{Rb}$, the gas used in Ref. [6]. Following the experiment we will take $\omega_{z}=2 \pi \times 11.6 \mathrm{~Hz}$ and $\omega_{\perp}=2 \pi \times 232 \mathrm{~Hz}$, and we will use $N g=10000$, which corresponds to a few times $10^{5}$ $\mathrm{Rb}$ atoms. For the small transverse deformation of the trap we have tried $\varepsilon=0.0,0.03$ and $0.06(\varepsilon=0.03$ is the closest one to the actual experiment [6]).

The norm, $N[\psi]=\int|\psi|^{2} d \mathbf{r}$, which is related to the number of bosons in the condensate, and the energy

$$
\begin{aligned}
E[\psi] & =\int \bar{\psi}\left[-\frac{1}{2} \Delta+V_{0}(\mathbf{r})+\frac{g}{2}|\psi|^{2}-\Omega L_{z}\right] \psi d \mathbf{r} \\
& =E_{0}(\psi)-\Omega L_{z}(\psi) .
\end{aligned}
$$

are conserved quantities. Each stationary solution of the GPE of the form $\psi_{\mu}(\mathbf{r}, t)=e^{-i \mu t} \phi(\mathbf{r})$, is a critical point of the energy (3) with the norm constraint, $\left.\frac{\partial E}{\partial \psi}\right|_{N}\left[\psi_{\mu}\right]=0$.

Critical frequencies.- Each state of the system is a "point" in the infinite-dimensional functional space on which the energy $E_{0}(\psi)$ and the angular momentum $L_{z}(\psi)$ are defined. In principle, due to the conservation of the energy, and due to the fact that dissipation at most drains energy out of the system, the minima of $E_{0}(\psi)$ are associated to stable states. Around these minima, the "motion" of the system is confined by energy barriers. However, rotation involves a pointwise change of the height of these infinite dimensional "surfaces" of energy, which move from $E_{0}(\psi)$ to $E_{0}(\psi)-\Omega L_{z}(\psi)$. This shift, which is stationary for each dynamical configuration of the trap adopted, can turn stable local minima into saddle points and open paths for the evolution of the condensate from simple nodeless state to states with one or more vortices.

Let us consider the case of an axially symmetric trap. These traps admit axially symmetric solutions with either no vortices, $\psi_{0}$, or with one centered vortex of integer charge, $\psi_{m} \propto \exp \{\operatorname{im} \arctan (y / x)\}$. In nonrotating traps, the vortexless state is actually the one with less energy. As the rotation speed is increased, the energies of the states with positive vorticity are all shifted down, $E_{m}(\Omega)=E_{m}(0)-m \Omega$, so that when $\Omega>\Omega_{m}=\frac{E_{m}(0)-E_{0}(\Omega)}{m}, m=1,2, \ldots$, the state $\psi_{m}$, i. e. a symmetric and straight vortex with vorticity $m$, is energetically more favorable than $\psi_{0}$. The values $\Omega_{m}$ are thus first estimates for the critical frequencies at which states with vorticity $m$ could be created.
We must note that the condition $\Omega>\Omega_{m}$, does not imply that $\psi_{m}$ is a global minimum but only that $E\left(\psi_{0}\right)>E\left(\psi_{m}\right)$. It is feasible for other states with the same vorticity to have less energy than the straight vortices. That is, another state $\tilde{\psi}_{m}$ might exist such that $E\left(\tilde{\psi}_{m}\right)<E\left(\psi_{m}\right)$. This is known to be the case for $\psi_{2}$ which is energetically less favorable than a pair of $m=1$ vortices 3, 3 . 4 .

In fact, for an axisymmetric vortex $\psi_{m}$ to be stable, it should be at least a local minimum of $E(\psi)$. One way of studying the local stability is by using linear stability analysis, i. e. we linearize the energy functional around any state $\delta(\mathbf{r})$

$$
E[\psi+\delta]=E[\psi]+E^{\prime}[\psi](\delta)+E^{\prime \prime}[\psi](\delta, \delta)+\mathcal{O}\left(|\delta|^{3}\right)
$$

and find a quadratic expansion

$$
E^{\prime \prime}[\psi](\delta, \delta)=\sum_{m, j} \lambda_{m, j}(\Omega ; \psi)\left|\delta_{m, j}\right|^{2},
$$

in which the perturbation is expanded on a suitable ba$\operatorname{sis} \phi_{m, j}(\rho, z) e^{i m \theta}$ 四] as $\delta(\mathbf{r})=\sum_{m, j} \delta_{m, j} \phi_{m, j}(\rho, z) e^{i m \theta}$. Following this procedure we are able to study the curvatures of the surface $(\psi, E[\psi])$ around that state — namely, the eigenvalues $\lambda_{m, j}(\Omega ; \psi)=\lambda_{m, j}(0 ; \psi)-m \Omega$ - This analysis allows us to decide when the operator $E^{\prime \prime}\left[\psi_{m}\right]$ is positive definite and consequently when a particular $\psi_{m}$ becomes a local minimum. This happens for a critical frequency that will be denoted by

$$
\bar{\Omega}_{\ell}=-\min _{j, m} \frac{\lambda_{m, j}\left(0 ; \psi_{\ell}\right)}{m-\ell} .
$$

For instance, when $\Omega=\bar{\Omega}_{1}$ the straight vortex becomes a local minimum.

To generate a condensate with vorticity two conditions must be satisfied. It is clear that the trap must rotate fast enough to make a vortex state energetically favorable, but it is also necessary that an energy decreasing path exists from $\psi_{0}$ to the vortex state, otherwise the transition could be inhibited due to the energy barrier around $\psi_{0}$ making this state metastable. Mathematically, there should exist a perturbation (a "direction" in the phase space), $\phi_{m, i}$, such that $\Omega>\lambda_{m, i}\left(0 ; \psi_{0}\right) / m$, a condition which looks similar to the bounds of Ref. [9]. We will define a destabilization frequency, given by

$$
\Omega_{M}=\min _{m, j}\left\{\frac{\lambda_{m, j}\left(0 ; \psi_{0}\right)}{m}\right\} .
$$

Within the interval $\Omega \in\left[0, \Omega_{M}\right)$ a condensate in a vortexless state cannot be forced to acquire angular momentum unless some additional energy is pumped in and the nodeless state $\psi_{0}$ could be metastable.

Results for symmetric vortices.- One could be tempted to think that the real situation is the simplest one, i. e. that by increasing $\Omega$ one should reach a point $\Omega_{1}$ in which 
$E\left(\psi_{0}\right)>E\left(\psi_{1}\right)$ and at the same time $\Omega_{1} \geq \bar{\Omega}_{1}, \Omega_{M}$. In this situation $\psi_{1}$ would become also a local minimum $\left(\bar{\Omega}_{1}\right)$ and the core state would loose its stability $\left(\Omega_{M}\right)$. However this is not the case, and the situation is more complicated as we will see below.

With the previous definitions in mind we have computed numerically the states $\psi_{0}, \psi_{1}, \ldots$ as well as the different frequencies $\Omega_{1}, \Omega_{2}, \Omega_{3}, \bar{\Omega}_{1}$ and $\Omega_{M}$. The results are plotted in Fig. 1 for a very elongated trap such as those used in the ENS experiment [6].

There are two relevant conclusions that may be obtained from this picture. The first one is that $\bar{\Omega}_{1}>$ $\Omega_{1}, \Omega_{2}$ which means that the ground state of the system may never be a symmetric vortex line. In other words, should it be energetically favorable for the ground state to acquire some vorticity, it will never be by means of straight vortex lines, but with some other structure. From a practical point of view, the large difference between $\Omega_{1}$ and $\bar{\Omega}_{1}$ also implies that the ENS experiment is working on a regime in which straight vortex lines are very hard to obtain. What are the new vortex structures which appear instead above $\Omega_{1}$ is a point we will discuss later.

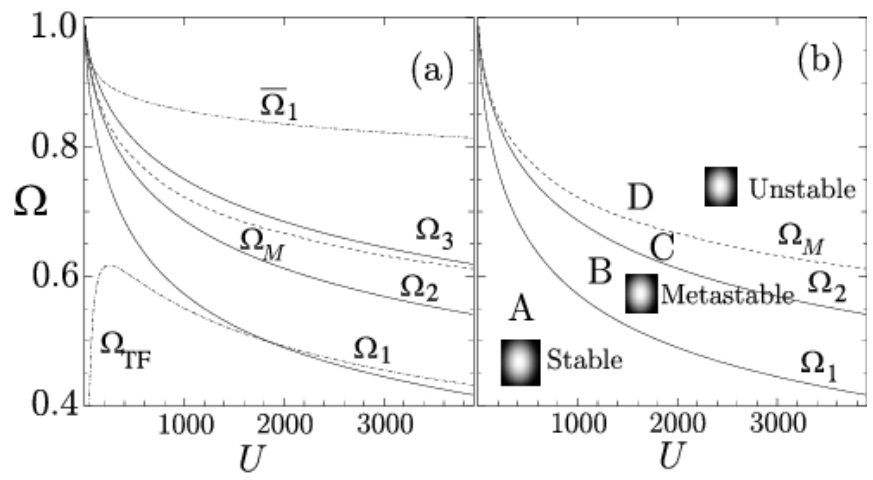

FIG. 1. (a) Critical angular speeds for an elongated condensate $\left(\omega_{z} / \omega_{\perp}=18.7\right)$ at different nonlinearities $\left(U=g N=4 \pi N a_{R b} / a_{\perp}\right)$. We plot the Thomas-Fermi estimate for the energy difference between a core and vortex state ( $\Omega_{T F}$, dash-dot line) the speeds $\Omega_{1}, \Omega_{2}$ and $\Omega_{3}$ at which vortices with charge $m=1,2,3$ become energetically favorable (solid lines), and the angular speed at which $\psi_{0}$ (vortexless state) becomes a saddle point $\left(\Omega_{M}\right.$, dashed line). (b) Regions with different phenomenology: from $\mathrm{A}$ to $\mathrm{C}$ there is an energy barrier surrounding the vortexless state; in $\mathrm{A}, \mathrm{B}$ and $\mathrm{C}$ the ground state has vorticity equal to 0,1 and 2 respectively; in $\mathrm{D}$ the energy barrier around the core state vanishes and more and more vortices become feasible.

The second and most relevant feature is that the vortexless state, $\psi_{0}$, remains a local minimum up to a speed, $\Omega_{M}$, which is much larger than the value at which vortices become energetically favorable (Regions B and $\mathrm{C}$ in Fig. 1(b)). Before reaching $\Omega=\Omega_{M}$ it is energetically expensive to introduce angular momentum into the condensate, since an energy barrier must be surpassed. For
$\Omega>\Omega_{M}$ states with one or more vortices become feasible at the same time. This persistent metastability of $\psi_{0}$ should be responsible for the experimental observation that a single angular speed is capable of producing states with a different number of vortex lines [6].

Our findings imply that once a vortex state is reached, the rotation speed may be ramped down and vortices should remain stable for some range of $\Omega$ values. For instance, if after reaching $\Omega_{M} \simeq 0.63 \omega_{\perp}$ for $U=g N=$ 3000 one gets a state with a vortex, then the rotation speed may be ramped down until $\Omega_{1} \simeq 0.46 \omega_{\perp}$ giving rise to a hysteresis phenomenon as it is graphically illustrated in Fig. 2(b).

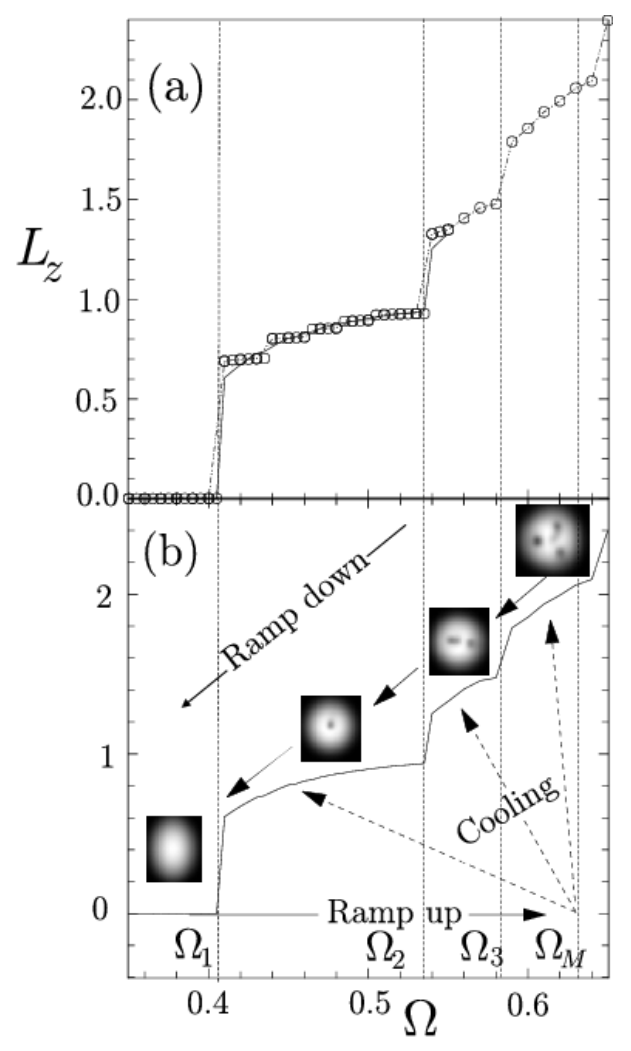

FIG. 2. (a) Angular momentum, $L_{z}$, of the ground state of the energy functional $E(\psi)$ [Eq. (3)] as a function of $\Omega$. Circles correspond to solutions over a grid with $32 \times 32 \times 64$ Fourier modes for $\varepsilon=0,0.03,0.06$. The solid line corresponds to the solutions on a grid with $64 \times 64 \times 128$ modes and $\varepsilon=0.03$. (b) Schematic picture of the angular momentum of the ground state versus angular speed, with a graphical description of the hysteresis mechanism. All figures are adimensional.

True ground states.- For $\Omega>\Omega_{M}$, or if the the energy barrier which surrounds the core state is overstepped, a condensate produced by means of evaporative cooling should correspond to the absolute minimum of the energy in the configuration space. We have worked numerically with the energy functional [Eq. (3)] in three spatial dimensions using a technique known as Sobolev 
gradients to find the ground states subject to some reasonable constraints - i. e. the norm and angular speed(simple minimization methods do not work for this problem). The details of the procedure are given in Ref. [11. We have applied this method on a Fourier basis with $32 \times 32 \times 64$ modes (enough for plotting purposes) and with $64 \times 64 \times 128$ modes (which is needed to lower the error in $L_{z}$ below $1 \%$.)

Figs. 2 and 3 summarize our results. In Fig. 3(a) we see that the ground state acquires vorticity when $\Omega$ is smaller than the core-state destabilization speed and the experimental values found in [6], in agreement with the predictions of the preceding paragraphs. Our numerical method also solves the question posed before: if the ground state must have some vorticity and it is not a straight vortex, what is its shape? In Fig. 3 we see that vortices are nucleated with deformed shapes. The combination of the sudden destabilization of the vortexless state, and the longitudinal deformations of vortex lines is enough to explain why vortex lines seem partially filled and why the angular momentum evolves almost continuously above $\Omega_{M}$. More details on the structure of bent vortices will be given elsewhere [12].

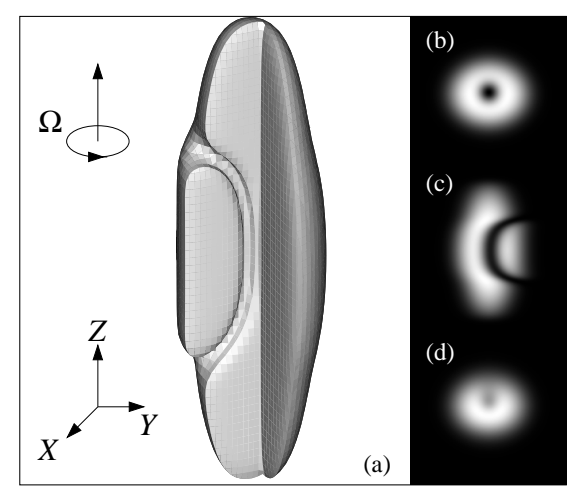

FIG. 3. Shape of the ground state of the Hamiltonian (3) for $\Omega=0.75 \omega_{\perp}, \gamma=18.7, N g=1000$ and $\varepsilon=0.06$. (a) Transverse section. (b) Density plot. One quarter of the condensate has been removed to allow the direct observation of the inner structure of the vortex line.

Discussion and conclusions.- We have used the GPE to study the nucleation of vortices in an elongated trap similar to that used at the ENS [6]. Our main prediction is that in such elongated traps there exists a mechanism which prevents the nucleation of vortices unless the condensate rotates much faster than the speed required to make one or more vortices energetically stable (metastabilization of $\psi_{0}$ ).

We predict that in such elongated traps, straight vortex lines are locally stable only for very large angular speeds - well beyond the values at which higher vorticites become preferable-. And we find the longitudinal deformation of these topological defects to be responsible for an almost continuous growth of the angular momen- tum with respect to the angular speed.

It is difficult to observe the bending of vortex lines in current experimental setups since condensates are expanded too much along their transverse dimensions. Nevertheless, other predictions of this paper such as the metastabilization phenomenon should be easily testable. First, before the destabilization of the vortexless state, $\Omega_{M}$, the vortex nucleation process is prevented, and the condensate may only adapt to the rotation speed by means of transverse deformations which are described in [13]. And second, a more controlled nucleation of vortices is possible by ramping up the trap beyond $\Omega_{M}$ and then carefully slowing down the condensate to some point above $\Omega_{1}$. An expansion of such cloud should lead to the observation of vortices for much lower values of $\Omega$ than those reported in Ref. [6].

This work has been partially supported by CICYT under grant PB96-0534.

[1] P. G. Saffman, "Vortex dynamics", Cambridge University Press (1997); Y. Kivshar, B. Luther-Davis, Phys. Rep. 298, 81 (1998).

[2] D. S. Rokhsar, Phys. Rev. Lett. 79 (1997) 2164; R. J. Dodd et al., Phys. Rev. A 56 (1997) 587; R. Dum, et al., Phys. Rev. Lett. 80 (1998) 2972; F. Zambelli, S. Stringari, Phys. Rev. Lett. 81 (1998) 1754; M. CaradocDavies et al., Phys. Rev. Lett 83, 895 (1999) T. Isoshima and K. Machida, Phys. Rev. A 59 (1999) 2203; D. L. Feder et al., Phys. Rev. Lett. 824956 (1999); M. R. Matthews et al., Phys. Rev. Lett. 832498 (1999); D. L. Feder et al., Phys. Rev. A 61011601 (2000); A. Svidzinsky and A. L. Fetter, Phys. Rev. Lett. 84, 5919 (2000).

[3] D. A. Butts and D. S. Rokhsar, Nature 397, 327 (1999).

[4] J. J. García-Ripoll and V. M. Pérez-García, Phys. Rev. A 60, 4864 (1999).

[5] M. R. Matthews, B. P. Anderson, P. C. Haljan, C. E. Wiemann, E. A. Cornell, Phys. Rev. Lett. 83 (1999) 2498; J. J. García-Ripoll, V. M. Pérez-García, Phys. Rev. Lett. 84, 4264 (2000); V. M. Pérez-García, J. J. García-Ripoll, Phys. Rev. A 62033601 (2000).

[6] K. W. Madison, F. Chevy, W. Wohlleben, J. Dalibard, Phys. Rev. Lett. 84806 (2000); F. Chevy, K. W. Madison, J. Dalibard, Phys. Rev. Lett. 85, 2223 (2000).

[7] A. A. Svidzinsky, A. L. Fetter, Phys. Rev. A, 62, 063617 (2000).

[8] D. L. Feder, A. A. Svidzinsky, A. L. Fetter, C. W. Clark, cond-mat/0009086

[9] F. Dalfovo, S. Stringari, e-print cond-mat/0009187

[10] F. Dalfovo et al., Rev. Mod. Phys. 71, 463 (1999).

[11] J. J. García-Ripoll, V. M. Pérez-García, e-print http://xxx.lanl.gov/abs/mat.AP/0008225.

[12] J. J. García-Ripoll and V. M. Pérez-García (preprint).

[13] J. J. García-Ripoll and V. M. Pérez-García, e-print condmat/003451. 